https://helda.helsinki.fi

\title{
Predictors of New Airway Obstruction - An 11 Year's Population-Based Follow-Up Study
}

\section{Vasankari, Tuula}

2019-01-02

Vasankari , T , Harkanen , T , Kainu , A , Sääksjärvi , K, Mattila , T, Jousilahti , P \& Laitinen , T 2019 , ' Predictors of New Airway Obstruction - An 11 Year's Population-Based

Follow-Up Study ' , COPD: Journal of chronic obstructive pulmonary disease , vol. 16 , no. 1 , pp. 45-50 . https://doi.org/10.1080/15412555.2019.1576163

http://hdl.handle.net/10138/308876

https://doi.org/10.1080/15412555.2019.1576163

acceptedVersion

Downloaded from Helda, University of Helsinki institutional repository.

This is an electronic reprint of the original article.

This reprint may differ from the original in pagination and typographic detail.

Please cite the original version. 
3 Tuula Vasankari, $\mathrm{MD}, \mathrm{PhD}$, Professor ${ }^{1,2}$

4 Tommi Härkänen, $\mathrm{PhD}^{3}$

5 Annette Kainu, MD, PhD ${ }^{4,5}$

$6 \quad$ Katri Sääksjärvi, $\mathrm{PhD}^{3}$

$7 \quad$ Tiina Mattila, $\mathrm{MD}, \mathrm{PhD}^{3,6}$

8 Pekka Jousilahti, MD, PhD, Professor ${ }^{3}$

$9 \quad$ Tarja Laitinen, $\mathrm{MD}, \mathrm{PhD}$, Professor ${ }^{1,7}$

$11{ }^{1}$ University of Turku, Division of Medicine, Department of Pulmonary Diseases and Clinical 12 Allergology, Turku University Hospital and University of Turku, PO Box 52 (Hämeentie 11), 1320521 Turku, Finland.

$14{ }^{2}$ Finnish Lung Health Association (FILHA), Filha ry, Sibeliuksenkatu 11 A 1, 00250 Helsinki, 15 Finland.

$16 \quad{ }^{3}$ National Institute for Health and Welfare, PO Box 30, 00271 Helsinki, Finland.

$17{ }^{4}$ University of Helsinki, Department of Medicine, 00014 University of Helsinki, Finland.

$18{ }^{5}$ Terveystalo Healthcare, Jaakonkatu 3B, 00100 Helsinki, Finland.

$19{ }^{6}$ Department of Pulmonary Diseases, Heart and Lung Center, Helsinki University Hospital, 20 Meilahti Triangle Hospital, $6^{\text {th }}$ floor, PO Box 372, 00029 HUS, Helsinki, Finland. 
23 Corresponding author: Tuula Vasankari, Finnish Lung Health Association (FILHA), Filha ry,

24 Sibeliuksenkatu 11 A 1, 00250 Helsinki, Finland.E-mail: tuula.vasankari@,filha.fi, tel.: +358 50 $25 \quad 5450589$.

26

27 
In the present study we aimed to investigate the incidence and predictors of spirometry based airway obstruction in a representative population-based sample.

Altogether 3863 subjects, 1651 males and 2212 females aged $\geq 30$ years had normal spirometry in year $2000.53 \%$ of them were never and $23 \%$ current smokers. A re-spirometry was performed 11 years later. Several characteristics, such as level of education, use of alcohol, physical activity, diet using AHEI index, BMI, circumwaist, sensitive CRP and cotinine of the laboratory values and comorbidities including asthma, allergic rhinitis, sleep apnoea and chronic bronchitis, as potential risk factors for airway obstruction were evaluated.

Using $\mathrm{FEV}_{1} / \mathrm{FVC}$ below the lower limit of normal, we observed 124 new cases of airway obstruction showing a cumulative 11 -year incidence of $3.2 \%$ and corresponding to an incidence rate of 5.6/1000 PY. The incidence rate was higher in men than in women (6.3/1000PY vs. 5.0/1000PY, respectively). The strongest risk factors were current smoking (OR 2.5) and previously diagnosed asthma (OR 2.1). Sensitive CRP associated with the increased risk and high AHEI index with the decreased risk of airway obstruction.

Using the similar study approach our findings on the incidence of airway obstruction are in line with the previously published figures in Europe. We were able to confirm the recent findings on the protective effect of healthy diet.

Keywords: COPD, airway obstruction, spirometry, incidence, healthy diet 


\section{Introduction}

Chronic obstructive pulmonary disease (COPD) is one of the leading causes of morbidity, disability and death worldwide [1,2]. Based on a global estimate in 2010 the prevalence of COPD in adults over 30 years of age was $11.4 \%$ being more prevalent among males $(14.3 \%)$ than females $(7.6 \%)$ [3].

In studies on COPD incidence, two main approaches have been used. First, diagnosis based studies have attempted to define COPD based on clinical outcomes such as outpatient contacts, hospital visits for exacerbations, or self-reported physician diagnosed COPD. These studies have reported incidence rates around 2.6-2.9 cases / 1.000 patient years (PY) with higher incidence rates found in males and among older age categories [4,5]. The second approach is based on by showing irreversible airway obstruction in spirometry. Furthermore, the definition of airway obstruction varies [6-12]. Many epidemiological studies have used the Global Initiative on Obstructive Lung Disease (GOLD) definition of a fixed ratio of forced expiratory volume in one second $\left(\mathrm{FEV}_{1}\right)$ to forced vital capacity (FVC) below 0.70 . Fixed ratio has the benefit of easy comparability between populations, but is known to give false positives in subjects over 50 years of age. One example of this is the Rotterdam study, the incidence was almost 50\% lower when population based reference values were used instead of the fixed ratio (5.5/1.000 PY vs. 8.9/1.000 PY), respectively [7].There is a large number of population based epidemiological studies that have evaluated the incidence of airway obstruction based on fixed ratio with widely varying incidence rates, 2.8-16.0 /1.000 PY [712]. Population age distribution, smoking habits and sampling differences can partially explain these widely varying incidence estimates.

Smoking is the main causative factor for COPD [2], but also other -causative and predisposing factors have been recognised. Many of them are linked to low socioeconomic status [13], occupational exposures [14] and old age [15]. Of studied biomarkers, sensitive C-reactive protein 
shows the most constant association to low lung function and COPD [16]. Recently the diet has become of great interest, especially the association between low vitamin D levels and declined lung function [17], even though no causality to the incidence of COPD could be observed [18]. Also an association has been observed between high consumption of processed meat and lower lung function [19], whereas high fruit and vegetable consumption lowered the risk of COPD among current and ex-smokers [20]. Lately, high Alternate healthy eating index (AHEI 2010) has been associated with a lower risk of COPD [21].

The primary objective of this study was to investigate the incidence of airway obstruction in a population with high standard of living and smoking steadily decreasing [22, 23]. Spirometry based incidence on airway obstruction have never been published in Finland. The secondary objective was to find out associative factors of development of airway obstruction.

\section{Methods}

A sample of 9922 subjects aged 18 years or over was drawn from the population register (two-stage stratified, random sample) and invited to a comprehensive health survey (Health 2000 survey) in 2000-2001 [24]. Of these subjects, 8028 (3637 males and 4391 females) were aged 30 years or over, and of whom altogether 6354 (79\%) subjects completed the health examination, donated blood samples, and performed spirometry.

All study subjects of Health 2000 survey who lived in Finland were invited to participate Health 2011 survey ( $\mathrm{n}=8135$ subjects), and 58.2\% (4736/8135) of them completed the health examination. The study population in the present study comprised of those 3863 subjects who were aged 30 years or over in Health 2000, participated and performed spirometry in both Health 2000 and Health 2011 surveys and had normal spirometry in Health 2000 survey. 
Our aim was to analyse how baseline data collected in Health 2000 survey predicted airway obstruction in follow-up until Health 2011 survey. In Health 2000 survey information on age and gender (female/male) were obtained from population register. Height, weight and circumwaist were measured. Body mass index (BMI) (weight $(\mathrm{kg}) /$ height $\left.^{2}\left(\mathrm{~m}^{2}\right)\right)$ was determined. The use of alcohol during previous 12 months was determined and categorised as: no, seldom (use of alcohol twice in a month or less) and often (use of alcohol once in a week or more often).

Smoking history was obtained through a standard interview and classified into three categories: never-smokers (those who had smoked daily less than 1 year during their life-time and quit smoking at least one month earlier), former smokers (those who had smoked daily over a year and quit smoking at least one month earlier) and current smokers (those who had smoked daily over a year and at least one cigarette, cigar or pipe for less than a month ago at any time preceding the survey). Educational level was categorised into three groups (basic, secondary, or higher). Leisure physical activity was classified into three groups: low (little physical exercise), moderate (exercise in connection with some hobbies or irregularly), or high (regular exercise).

Subjects were considered asthmatic if they reported that they had been diagnosed with asthma by a physician, that they were currently in a physician's control and received medication for asthma. The history, symptoms and possible findings of allergic rhinitis, chronic bronchitis, and sleep apnoea were inquired about in the basic questionnaire and categorised as having these or not.

Participants' habitual dietary intake over preceding 12 months was collected with a validated 128item semi quantitative food frequency questionnaire (FFQ) $[25,26]$. Adherence to the healthy diet was measured using the Alternate Healthy Eating Index (AHEI) developed by McCullough et al. (2002) [27]. The AHEI in our data included the intake of seven components (vegetables; fresh fruits and berries; nuts and legumes; rye; the ratio of white to red meat; the ratio of polyunsaturated to saturated fat; trans fat), which were divided into sex-specific quintiles. The first six components 
received scores in an ascending order, so that one point was assigned for intakes in the lowest quintile and five points for intakes in the highest quintile. The trans fats component received scores in the descending order, with the lowest quintile gaining a value of five points and the highest quintile a value of one point. The total score ranged from 7 (worst) to 35 (best), with higher score values representing greater adherence to a healthy diet. Total energy in take was measured in kilocalories (kcal) according to the FFQ.

Serum samples used to characterise the participants were collected and frozen at $-20{ }^{\circ} \mathrm{C}$ and stored at $-70{ }^{\circ} \mathrm{C}$. Cotinine concentrations were determined by a modified method of the Nicotine Metabolite RIA kit (Diagnostic Products Corporation, LA, USA). For cotinine a cut-off point of $100 \mu \mathrm{g} / \mathrm{L}$ or over was used to separate smokers from non-smokers. The level of sensitive $\mathrm{C}$ reactive protein (sCRP) was determined using a chemiluminescent immunometric assay (Immulite, Diagnostic Products Corporation, Los Angeles, CA, USA).

In Health 2000, Vitalograph bellow spirometers (Vitalograph Ltd., Buckingham, England) were used, while in Health 2011 the Medikro ${ }^{\circledR}$ SpiroStar flow-volume spirometer and Medikro ${ }^{\circledR}$ Spiro2000 software, was used. To ensure comparability between the measurements a validation study was performed, which showed no significant difference between the two devices [24]. The spirometers were calibrated with a one-litre calibration pump, and the equipment was checked every morning before the measurements. The measurements were made by specially trained laboratory technicians following standard guidelines and instructions. The technicians demonstrated the test procedure to all subjects individually. When the subject had learned the technique and rehearsed it once or twice, the aim was to produce at least two as consistent curves as possible. For forced expiratory volume in one second $\left(\mathrm{FEV}_{1}\right)$ and forced vital capacity $(\mathrm{FVC})$ the technician instructed the subjects to fill their lungs with air and then to exhale as forcefully and completely as possible, urging them towards the end of the test. Failed efforts and those with questionable performance due to fatigue, or otherwise poor co-operation were excluded from the analysis. The quotient $\mathrm{FEV}_{1} / \mathrm{FVC}$ 
was calculated using the highest readings of $\mathrm{FEV}_{1}$ and $\mathrm{FVC}$ from technically acceptable efforts recorded in BTPS (body temperature and pressure, saturated with water vapour) values. $\mathrm{FEV}_{1} / \mathrm{FVC}$ below the lower limit of normal $(<\mathrm{LLN})$ was considered to indicate airway obstruction.

Bronchodilation was not performed for all subjects. The individual results were computed on the basis of the GLI reference values [6] for corresponding age, sex and height were used. Development of a new airway obstruction was analysed from the spirometry performed in Health 2011.

Health 2000 study was approved by the Ethics Committee for Epidemiology and Public Health in the Hospital District of Helsinki and Uusimaa and Health 2011 in the Coordinating Ethics Committee of the Hospital District of Helsinki and Uusimaa. All participants gave their written informed consent.

The sampling design was accounted for in all analyses using the survey package $[28,29]$ of the $R$ statistical software package [R Core Team (2017). R: A language and environment for statistical computing. R Foundation for Statistical Computing, Vienna, Austria. URL https://www.Rproject.org/]. The effects of oversampling of the 80 -year old and older in 2000, and nonresponse in the full sample were accounted for using the inverse probability weighting [30].

Regression analyses were based on generalised linear models. In addition to the simple regression models containing the risk factors one at a time, in which only age and sex were adjusted for (model 1), we adjusted also for age, sex and level of education (model 2), for age, sex and smoking (model 3), for age, sex, level of education and smoking (model 4) and also a fully adjusted multiple regression model containing all aforementioned risk factors (model 5) was analysed. Crude incidence rates and their $95 \%$ confidence intervals were calculated per 1000 person years.

\section{Results}


There were altogether 3863 subjects, 1651 (42.7\%) males and $2212(57.3 \%)$ females, with normal spirometry during the first survey and a re-performed spirometry 11 years later. Baseline characteristics in the study population including demographics, physical examinations, tested laboratory values and patient reported outcomes at baseline are described in Table 1. The prevalence of nonresponders who participated in Health 2000 but not in Health 2011 was biggest in subjects aged 65-100 in Health 2000.

New cases of airway obstruction occurred in 124 subjects, with a cumulative 11-year incidence of $3.2 \%$ corresponding to an incidence rate of 5.6/1000 person-years (PY) (95\% CI 3.8-5.6) (Table 2). The incidence rate of males was higher (6.3/1000PY, 95\% CI 4.9-8.2) and that of females (5.0/1000PY, 95\% CI 3.9-6.6). The highest incidence rate, 9.9/1000PY (95\% CI 4.8-16.7), was in the age group of $65-100$ years.

The development of airway obstruction associated in fully adjusted multiple regression model with current smoking, previously diagnosed asthma, increasing sCRP and decreasing AHEI index, and of these current smoking and previously diagnosed asthma were the strongest risks for airway obstruction (Table 1). In basic model with age and sex adjustment development of airway obstruction associated also with low physical activity, chronic bronchitis, decreasing BMI and waist circumference, and additionally, decreasing AHEI index meaning healthy diet protected from airway obstruction whereas the total energy intake did not have an effect. Cotinine had no association with airway obstruction in fully adjusted model (not reported). Cotinine and selfreported smoking are indicators of same phenomenon taking power in analysis from each other, though cotinine $\geq 100 \mu \mathrm{g} / \mathrm{mL}$ had an association with airway obstruction in other models (Table 1).

\section{Discussion}


In this study the incidence of airway obstruction based on spirometry ( $\mathrm{FEV}_{1} / \mathrm{FVC}$ below the lower limit of normal) was evaluated in a population based cohort (age $>30$ years of age) with a followup of 11 years. The overall incidence rate was 5.6/1000 PY, 6.3/1000 PY for males and 5.0/1000 PY for females. The main determinants for the development of airway obstruction were current smoking, previously diagnosed asthma, increased sCRP and low AHEI index.

In previous studies the incidence rates of COPD have varied from 2.6-16.0 per $1000 \mathrm{PY}$ [4,5,7-12]. The wide variation can be at least partially explained by differences in study approaches. When the incidence rates are based on retrospective health registries, such as given diagnoses, hospital admission data or use of medication, the data is highly dependent on the probability of a patient seeking for medical help, the diagnostic practices of different kind of health-care facilities as well as the coverage of register data. Studies evaluating self-reported physician-diagnosed COPD, symptoms or medications indicative of COPD are dependent on the participants' understanding on his or her health.

When the estimates are based on spirometry data, it is possible to find also the undiagnosed cases. Spirometry based incidence studies are also more comparable to each other, even though the definition of the cut-off value for airway obstruction can vary. Fixed $\mathrm{FEV}_{1} / \mathrm{FVC}$-ratio used frequently in many epidemiological studies is naturally lower in tall subjects and reduces with normal ageing. In the present study we used the lower limit of normal (LLN) which is individually predicted to determine airway obstruction in comparison to age and body size match healthy, nonsmoking population. These estimates do not cause age or size related bias in the results. In the Rotterdam Study, the pre-bronchodilator $\mathrm{FEV}_{1} / \mathrm{FVC}<0.70$ or in the absence of spirometry, COPD diagnosis verified from medical records, resulted in an incidence rate of 8.9/1000 PY. Based on spirometry data alone the incidence rate of $11.7 / 1000 \mathrm{PY}$ was observed with fixed ratio and much lower incidence of 5.2/1000PY with LLN criteria [7]. This was in line with our results. In the 
215 Netherlands, however smoking was more prevalent. Only 35\% of subjects were non-smokers, 216 compared to $53 \%$ in the Finnish population [7].

217 Current smoking and previous diagnosis of asthma associated independently to incident airway obstruction, both well recognised risk factors of COPD [2]. Smoking had decreased in the Health 2192000 survey significantly during the follow-up time of 11 years, among the men in all age cohorts 220 and among the women in age cohorts of 30-54 years [23]. The earlier finding of an association 221 between sCRP and COPD was also confirmed in this study [16].

222 The protective effect of a healthy diet has been under recent research interest. In this study, an 223 inverse association between AHEI index and incident, spirometry based airway obstruction was 224 observed. The association reminded significant in all models performed which means healthy diet 225 protected from airway obstruction regardless of energy intake. High AHEI index reflects the 226 protective effect of high intakes of whole grains, polyunsaturated fatty acids, nuts and long chain 227 omega-3 fats and low intakes of red/processed meats, refined grains and sugar sweetened drinks. 228 Earlier studies have reported that high fruit and vegetable consumption [20] and more-frequent fish 229 intake $[26,31]$ can protect from the disease. AHEI score has been studied in clinical COPD only 230 once and an inverse association between higher AHEI score and the incidence of COPD was 231 observed [21]. In this large prospective study diagnosis of COPD was based on self-reported 232 questionnaire. As a validation study, a random sample of medical records of the participants were 233 assessed. In $71 \%$ of the COPD cases spirometry was available confirming the self-reported COPD $234[21]$.

235 In addition to COPD, chronic bronchitis is a symptom of multiple other conditions [32]. Therefore, 236 our result about chronic bronchitis having association with new airway obstruction only in age and 237 sex adjusted model is rational. In BOLD study $129(11 \%)$ subjects had both chronic bronchitis and 
COPD [32], while we found that $9(7.6 \%)$ of those with new obstruction reported symptoms of chronic bronchitis; these results being comparable.

A limitation in our study is that bronchodilation test was not performed for all, and therefore, we could not separate reversible and non-reversible airway obstruction. However, our study population was aged 30 and over at baseline and only 14 subjects (11.2\%, Table 1$)$ with new airway obstruction reported to have asthma in Health 2011, and therefore apparently most of these with new airway obstruction are COPD [33]. Values of the risk factors, such as smoking status, sCRP and AHEI index, might have changed during follow-up and have some effect on our results, but as our objective was to assess the potential predictors of obstruction incidence we did not evaluate the associations of risk factor changes during the follow-up and the obstruction incidence. Furthermore, these associations are likely to operate in two directions as, for example, weight changes according to the lifestyle factors (daily energy intake and physical activity). Additionally, we had no data about smoked pack-years and respiratory medications used in pulmonary diseases. The follow-up time of 11 years might be, especially, in the younger age groups too short for the development of airway obstruction, and correspondingly, in older age groups too long. Additionally, the prevalence of nonresponders was biggest in the oldest age group, aged 65-100 in Health 2000 and 76 or older in Health 2011, where the physical fragility probably affected why these subjects performed not spirometry or participated in Health 2011 at all, however, this was accounted for in analyses by inverse probability weighting.

\section{Conclusions}

Using the similar study approach our findings on the incidence of airway obstruction are in line with the previously published figures in Europe. Our study nicely completed the previous findings 
261 and further addressed the importance of healthy diet as an independent risk factor for the

262 development of airway obstruction.

264 The authors report no conflicts of interest.

\section{References}

2. Fletcher C, Peto R. The natural history of chronic airflow obstruction. BMJ. 1977; 1(6077):1645-8.

3. Adeloye D, Chua S, Lee C, Basquill C, Papana A, Theodoratou E, Nair H, Gasevic , Sridhar D, Campbell H, Chan KY, Sheikh A, Rudan I; Global Health Epidemiology Reference Group (GHERG). Global and regional estimates of COPD prevalence: Systematic review and meta-analysis. J Glob Health. 2015; 5(2): 020415.

4. Afonso ASM, Verhamme KMC, Sturkenboom MCJM, Bruselle GGO. COPD in the general population: prevalence, incidence and survival. Resp Med. 2011 (105): 1872-84.

5. Garcia Rodriguez LA, Wallander MA, Tolosa LB, Johansson S. Chronic obstructive pulmonary disease in UK primary care: incidence and risk factors. COPD. 2009; 6 (5): 36979.

6. Quanjer PH, Stanojevic S, Cole TJ, Baur X, Hall GL, Culver BH, Enright PL, Hankinson JL, Ip MSM, Zheng J, Stocks J and the ERS Global Lung Function Initiative. ERS Task 
Force. Multi-ethnic reference values for spirometry for the 3-95-yr age range: the global lung function 2012 equations. Eur Respir J 2012; 40 (6): 1324-1343.

7. Terzikhan N, Verhamme KMC, Hofman A, Stricker BH, Brusselle GG, Lahousse L. Prevalence and incidence of COPD in smoker and non-smokers: the Rotterdam study. Eur J Epidemiol. 2016; 31 (8): 785-92.

8. Van Durme YM, Verhamme KM, Stijnen T, Van Rooij FJA, Van Pottelberge GR, Hofman A, Joos GF, Stricker BHC, Brusselle GG. Prevalence, incidence, and lifetime risk for the development of COPD in the elderly: the Rotterdam study. Chest. 2009; 135 (2): 368-77.

9. Kojima S, Sakakibara H, Motani S, Hirose K, Mizuno F, Ochiai M, Hashimoto S. Incidence of chronic obstructive pulmonary disease, and the relationship between age and smoking in a Japanese population. J Epidemiol 2007; 17(2): 54-60.

10. De Marco R, Accordini S, Cerveri I, Corsico A, Antó JM, Künzli N, Janson C, Sunyer J, Jarvis D, Chinn S, Vermeire P, Svanes C, Ackermann-Liebrich U, Gislason T, Heinrich J, Leynaert B, Neukirch F, Schouten JP, Wjst M, Burney P. Incidence of chronic obstructive pulmonary disease in a cohort of young adults according to the presence of chronic cough and phlegm. Am J Respir Crit Care Med. 2007; 175 (1):32-9.

11. Lindberg A, Eriksson B, Larsson L-G, Rönmark E, Sandström T, Lundbäck B. Seven-year cumulative incidence of COPD in an age-stratified general population sample. Chest. 2006; 129 (4):879-85.

12. Johannessen A, Omenaas E, Bakke P, Gulsvik A. Incidence of GOLD-defined chronic obstructive pulmonary disease in a general adult population. Int J Tuberc Lung Dis. 2005; 9 (8): 926-32.

13. Johannessen A, Eagan TM, Omenaas ER, Bakke PS; Gulsvik A. Socioeconomic risk factors for lung function decline in a general population. Eur Respir J. 2010; 36 (3): 480-7. 
14. Mehta AJ, Miedinger D, Keidel D, Bettschart R, Bircher A, Bridevaux PO, Curjuric I, Kromhout H, Rochat T, Rothe T, Russi EW, Schikowski T, Schindler C, Schwartz J, Turk A, Vermeulen R, Probst-Hensch N, Künzli N; SAPALDIA team. Occupational exposure to dusts, gases, and fumes and incidence of chronic obstructive pulmonary disease in the Swiss cohort study on air pollution and lung and heart diseases in adults. Am J Respir Crit Care Med. 2012; 185 (12): 1292-300.

15. Ito K, Barnes PJ. COPD as a disease of accelerated lung aging. Chest. 2009; 135 (1): 17380.

16. Agassandian M, Shurin G, Ma Y, Shurin MR. C-reactive protein and lung diseases. Int J Biochem Cell Biol. 2014; 53: 77-88.

17. Black PN, Scragg R. Relationship between serum 25-hydroxyvitamin D and pulmonary function in the third national health and nutrition examination survey. Chest. 2005; 128 (66): $3792-8$.

18. Kunisaki KM, Niewoehner DE, Singh RJ, Connett JE. Vitamin D status and longitudinal function decline in the Lung Health Study. Eur Respir J. 2011; 37 (2): 238-43.

19. Okubo H, Shaheen SO, Nitani G, Jameson KA, Syddall HE, Sayer AA, Dennison EM, Cooper C, Robinson SM; Hertfordshire Cohort Study Group. Processed meat consumption and lung function: modification by antioxidants and smoking. Eur Respir J. 2014; 43 (4): $972-82$.

20. Kaluza J, Larsson SC, Orsini N, Linden A, Wolk A. Fruit and vegetable consumption and risk of COPD: a prospective cohort study of men. Thorax. 2017; 72 (6): 500-9.

21. Varraso R, Chiuve SE, Fung TT, Barr RG, Hu FB, Willett WC, Camargo CA. Alternate Healthy Eating Index 2010 and risk of chronic obstructive pulmonary disease among US women and men: prospective study. BMJ. 2015; 350: h286. 
22. Kainu A, Pallasaho P, Pietinalho A. No change in prevalence of symptoms of COPD between 1996 and 2006 in Finnish adults - a report from the FinEsS Helsinki Study. Eur Clin Respir J 2016; 3: 31780.

23. Koskinen S, Lundqvist A, Ristiluoma N, eds. Health, functional capacity and welfare in Finland in 2011. National Institute for Health and Welfare (THL), Report 68/2012. Helsinki 2012. ISBN 978-952-245-769-1.

24. Heistaro S. (ed), Methodology report. Health 2000 Survey. Publications of the National Public Health Institute B 26/2008. National Public Health Institute, Helsinki, Finland 2008, ISBN 978-951-740-860-8 (pdf), http://www.terveys2000.fi.

25. Männistö S, et al. Reproducibility and validity of a food frequency questionnaire in a casecontrol study on breast cancer. J Clin Epidemiol. 1996; 49:401-9.

26. Paalanen L, Männistö S, Virtanen MJ, Knekt P, Räsänen L, Montonen J, Pietinen P. Validity of a food frequency questionnaire varied by age and body mass index. J Clin Epidemiol. 2006 Sep;59(9):994-1001.

27. McCullough ML, Feskanich D, Stampfer MJ, et al. Diet quality and major chronic disease risk in men and women: moving toward improved dietary guidance. Am J Clin Nutr. 2002;76: $1261-71$

28. Lumley T. Analysis of complex survey samples. J Stat Software. 2004; 9(1): 1-19.

29. Lumley T. Survey: analysis of complex survey samples. 2016. R package version 3.31-5.

30. Härkänen T, Karvanen J, Tolonen H, Lehtonen R, Djerf K, Juntunen T, Koskinen S. Systematic handling of missing data in complex study designs - experiences from the Health 2000 and 2011 Surveys. Journal of Applied Statistics.2016; 43 (15): 2772-90.

31. Varraso R, Barr RG, Willett WC, Speizer FE, Camargo CA Jr. Fish intake and risk of chronic obstructive pulmonary disease in 2 large US cohorts. Am J Clin Nutr. 2015; 101 (2): 354-61. 

P, Nizankowska-Mogilnicka E, Burney PGJ; BOLD collaborators; BOLD study collaborators. Prevalence and burden of chronic bronchitis symptoms: results from the BOLD study. Eur Respir J. 2017; 22;50(5).

33. GINA Report, Global strategy for asthma Management and Prevention. Available at: https://ginasthma.org/2018-gina-report-global-strategy-for-asthma-management-andprevention/. Last accessed 27.11.2018. 